\title{
Electronic structures of finite-length carbon nanotubes: Crossover from fullerenes to nanotubes
}

\author{
Susumu Okada \\ Institute of Physics and Center for Computational Sciences, \\ University of Tsukuba, Tennodai, Tsukuba 305-8571, Japan and \\ CREST, Japan Science and Technology Agency, \\ 4-1-8 Honcho, Kawaguchi, Saitama 332-0012, Japan
}

(Dated: December 26, 2006)

\begin{abstract}
Finite-size effects in armchair $(n, n)$ carbon nanotubes are studied as a function of the tube length by using the generalized tight-binding calculations. End structures of the tubes considered here are caps formed from hemispherical pieces of $I_{h}$ fullerenes and hydrogen-free open ends. It has been clarified that the dimensionality in electronic structures of the finite-length nanotubes only depends on an aspect ratio of the tube diameter to the length of the cylindrical region. The aspect ratio where the finite-length tubes exhibit one-dimensional properties is found to be about four. The results corroborate that the nanotubes with their length of $10 \mathrm{~nm} \sim 100 \mathrm{~nm}$ experimentally observed could be regarded as one-dimensional electron systems.
\end{abstract}

PACS numbers: 73.22.-f, 71.20.Tx, 73.20.At 


\section{INTRODUCTION}

Following discoveries of fullerene [1] and carbon nanotube [2], there have been a lot of experimental and theoretical studies on these nanometer-scale materials [3]. Although, these materials are known to consist of $\mathrm{C}$ atoms with three-fold coordination as in the case of the graphite, their global network topologies are vastly different from that of the graphite: The fullerenes possess hollow-cage structures with zero-dimension due to existence of twelve pentagons. The nanotubes possess one-dimensional structures obtained by rolling up a graphene sheet into a tubular form. These peculiar global network topologies significantly result in interesting variations in their electronic properties. In the fullerenes, it has been clarified that the electronic structure is determined by the size and symmetry of the carbon cage [4]. On the other hand, in the nanotubes, early theoretical studies have revealed that $(n, m)$ nanotubes are metal when the remainder of $|n-m|$ divided three is zero, and that they are semiconducting otherwise [5-7].

Although both fullerene and nanotube are the three-fold coordinate networks, their electronic properties have been individually studied due to the different dimensionality of their networks. However, we can regard the nanotubes as a one-dimensional limit of the cylindrical fullerenes such as $\mathrm{C}_{70}$ and the fullerene as a zero-dimensional limit of the nanotubes. Thus, they are representative of two different limits of the nanometer-scale carbon materials. Based on this point of view, they should have critical lengths in which the electronic properties exhibit a crossover from one-dimensional character to the zero-dimensional one and vice versa.

Extractable large fullerenes are known to possess lower symmetries such as $C_{2}$ and $C_{1}$ in spherical shapes [8]. Indeed, large fullerenes in cylindrical or ellipsoidal shape have rarely been extracted in the conventional synthesis process so far. However, recent transmission electron microscope (TEM) images clarified that coalescence of $\mathrm{C}_{60}$ molecules encapsulated in nanotubes (carbon peapods) results in the cylindrical carbon nano-capsule with the length of about $10 \mathrm{~nm}$ [9]. Furthermore, it was also found that finite length nanotubes with a few tenths nm are synthesized on several catalysts, e.g. Cerium and Yttrium, by using an arc-discharge method $[10,11]$. In the field of the engineering, nanotubes with their length ranging from $10 \mathrm{~nm}$ to $100 \mathrm{~nm}$ are expected to be applicable for the nanometerscale electronic devices as a quantum wire. In the scientific viewpoint, they are a missing 
ring between fullerenes and nanotubes, and seamlessly connect these two nano-materials possessing different dimensionality. Thus, it is of interest whether the electronic structures of these materials exhibit one-dimensional character or not.

There have been a lot of theoretical calculations those studied the electronic structure of the cylindrical fullerenes [12-18]. However, due to a large number of carbon atoms in nano-capsules experimentally observed, the electronic structures of the capsules with their length of $10 \mathrm{~nm}$ (finite-length nanotube) have never been elucidated yet. Thus, in the present work, to evaluate the critical length that gives the crossover from zero-dimensional to one-dimensional electronic properties of the finite-length nanotubes, we calculate the electronic structure of the nanotubes with their lengths up to $10 \mathrm{~nm}$ by using the generalized tight-binding model which quantitatively reproduces electronic structures of various carbon materials. Here, $(5,5)$ and $(10,10)$ nanotubes are studied as representatives and salient characteristics of the finite-length nanotubes are elucidated. For each nanotube, we consider two different conditions of its ends; (i) both ends are capped by the hemisphere of $\mathrm{C}_{60}$ for $(5,5)$ and $\mathrm{C}_{240}$ for $(10,10)$ to simulate the nanocapsules synthesized inside the nanotube, (ii) one of two ends is capped while the other is opened to simulate the nanotubes grown on the catalysts. Our calculations corroborate that the electronic structures of the finite-length nanotubes experimentally observed can be regarded as one-dimensional material exhibiting the characteristics of the electronic structures of infinite-length nanotubes. Furthermore, the critical length is found to exhibit a universal feature for all nanotubes studied here, which are determined by the aspect ratio of the nanotubes.

\section{CALCULATION METHODS}

The electronic structures of nanotubes are calculated by using the generalized tightbinding model $[19,20]$. Here, $2 s$ and $2 p$ states of a carbon atom are used as the basis set to discuss the $\pi$ - $\sigma$ rehybridization induced by the curvature of the nanotube and the cap. Furthermore, the nonorthogonality of the atomic orbitals between neighboring sites is fully taken into account. Transfer, $t_{i, j}^{\alpha, \beta}$, and overlap integrals, $s_{i j}^{\alpha, \beta}$, between neighboring atomic orbitals have suitable distance dependences in order to reproduce the electronic band structures of various atomic configuration of the carbon atoms. Here we adopt following four 
types of the transfer integrals of the Slater-Koster type between two C atomic sites:

$$
\begin{aligned}
& t_{a b}^{s s \sigma}(r)=-7 \sqrt{v_{a}^{s} v_{b}^{s}} R^{s s \sigma}\left(4 r /\left(r_{s}^{a}+r_{s}^{b}\right)\right) Q(r), \\
& t_{a b}^{s p \sigma}(r)=-7 \sqrt{v_{a}^{s} v_{b}^{p \sigma}} R^{s p \sigma}\left(4 r /\left(r_{s}^{a}+r_{p \sigma}^{b}\right)\right) Q(r), \\
& t_{a b}^{p p \sigma}(r)=-7 \sqrt{v_{a}^{p \sigma} v_{b}^{p \sigma}} R^{p p \sigma}\left(4 r /\left(r_{p \sigma}^{a}+r_{p \sigma}^{b}\right)\right) Q(r), \\
& t_{a b}^{p p \pi}(r)=-7 \sqrt{v_{a}^{p \pi} v_{b}^{p \pi}} R^{p p \pi}\left(4 r /\left(r_{p \pi}^{a}+r_{p \pi}^{b}\right)\right) Q(r),
\end{aligned}
$$

where $a$ and $b$ denote atomic species. Values of the parameters for the $\mathrm{C}$ atom used in the present work are given as follows; $v^{s}=6.6 \mathrm{eV}, v^{p \sigma}=4.3 \mathrm{eV}, v^{p \pi}=4.5 \mathrm{eV}, r_{s}=0.620 \AA$, $r_{p \sigma}=0.810 \AA$, and $r_{p \pi}=0.550 \AA$. The origin of energy is defined as the $2 p$ level energy of the $\mathrm{C}$ atom. The orbital energy of the $2 s$ level is taken to be $7 \mathrm{eV}$ lower than that of the $2 p$ level. Similarly, the overlap integrals are given as follows

$$
\begin{aligned}
& s_{a b}^{s s \sigma}(r)=7 \sqrt{u_{a}^{s} u_{b}^{s}} R^{s s \sigma}\left(4 r /\left(r_{s}^{a}+r_{s}^{b}\right)\right) Q(r), \\
& s_{a b}^{s p \sigma}(r)=7 \sqrt{u_{a}^{s} u_{b}^{p \sigma}} R^{s p \sigma}\left(4 r /\left(r_{s}^{a}+r_{p \sigma}^{b}\right)\right) Q(r), \\
& s_{a b}^{p p \sigma}(r)=7 \sqrt{u_{a}^{p \sigma} u_{b}^{p \sigma}} R^{p p \sigma}\left(4 r /\left(r_{p \sigma}^{a}+r_{p \sigma}^{b}\right)\right) Q(r), \\
& s_{a b}^{p p \pi}(r)=7 \sqrt{u_{a}^{p \pi} u_{b}^{p \pi}} R^{p p \pi}\left(4 r /\left(r_{p \pi}^{a}+r_{p \pi}^{b}\right)\right) Q(r),
\end{aligned}
$$

where $u^{s}=u^{p \sigma}=u^{p \pi}=1 / 7 \mathrm{eV}$. The functions $R^{s s \sigma}$ etc. are defined as

$$
\begin{aligned}
& R^{s s \sigma}(x)=e^{-x}\left(1+x+\frac{x^{2}}{3}\right), \\
& R^{s p \sigma}(x)=-e^{-x}\left(x+\frac{x^{2}}{3}\right), \\
& R^{p p \sigma}(x)=-e^{-x}\left(-1+x+\frac{x^{2}}{3}\right), \\
& R^{p p \pi}(x)=e^{-x}\left(1+x+\frac{x^{2}}{3}\right) .
\end{aligned}
$$

We use a smooth cutoff function $\mathrm{Q}(\mathrm{r})$;

$$
Q(r)= \begin{cases}1 & \left(\text { for } r<r_{1}\right) \\ \frac{1}{2}\left(1+\cos \frac{\pi\left(r-r_{1}\right)}{r_{2}-r_{1}}\right) & \left(\text { for } r_{1}<r<r_{2}\right) \\ 0 & \left(\text { for } r_{2}<r\right) .\end{cases}
$$

The values of cutoff parameters $r_{1}$ and $r_{2}$ used here are 3.6 and $4.0 \AA$, respectively. All the parameters are chosen to give consistent results with those by the LDA calculation for both graphite and solid $\mathrm{C}_{60}$ [20]. Indeed, the present model is found to be accurate enough to reproduce the electronic structure of various carbon materials $[4,5]$. 
(a)

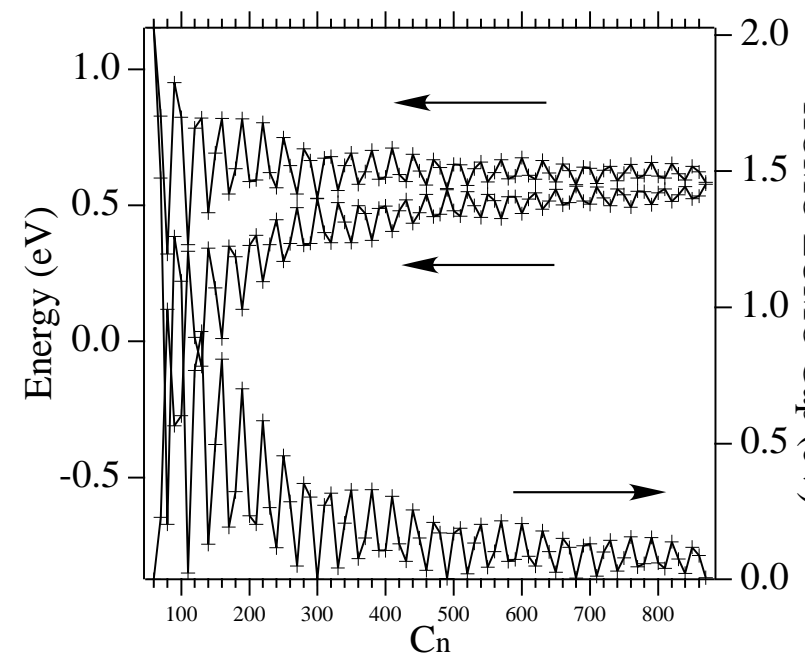

(b)

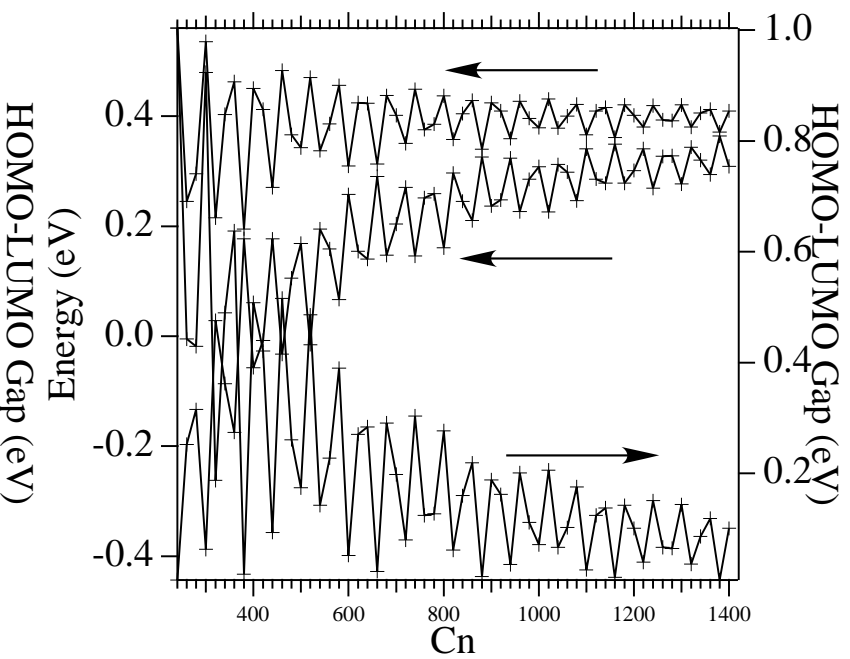

FIG. 1: Energies of HO and LU states and energy gap between HO and LU states for (a) $(5,5)$ nanotubes with $\mathrm{C}_{60}$ caps and (b) $(10,10)$ nanotubes with $\mathrm{C}_{240}$ caps.

\section{RESULTS AND DISCUSSIONS}

First, we discuss the electronic structures near the energy gap of the finite-length $(5,5)$ and $(10,10)$ nanotubes of which ends are capped by hemispheres of $I_{h}$ fullerenes of $\mathrm{C}_{60}$ and $\mathrm{C}_{240}$, respectively. As shown in Fig. 1, eigenvalue of the highest occupied (HO) and the lowest unoccupied (LU) states oscillate in nearly triple periodicity of the nanotube unit. Owing to this oscillation, the energy gap between HO and LU states also oscillates in the same period. Furthermore, the energy gap asymptotically decreases with increasing the tube length. The oscillation is interpreted that the length of the tubular region results in the set of discretized wave number along the one-dimensional energy band of the armchair nanotube. The discretization results in the triple periodicity for their energy level. On the other hand, we find that the energy gaps of both nano-capsules exhibit incommensurability for the triple periodicity. A recent density-functional study also demonstrated the incommensurability in the tube-like fullerenes up to $\mathrm{C}_{180}$ [16]. It should be noted that the incommensurability does not take place in $\pi$ tight-binding calculations. Thus, the incommensurability of the oscillation is caused by the curvature effects at the caps and the cylindrical part of the nano-capsule induced by the $\pi$ and $\sigma$ rehybridization.

Next, we show the density of states (DOS) of the finite-length nanotubes. Figure 2 shows 
(a)

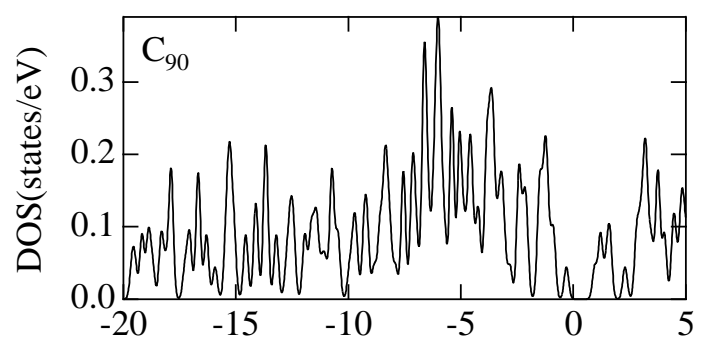

(b)

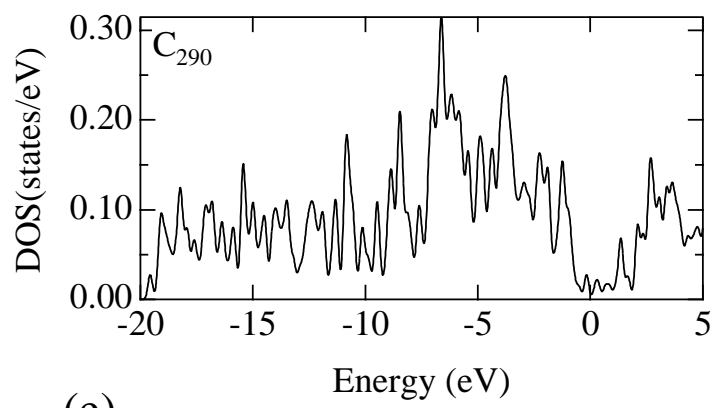

(e)

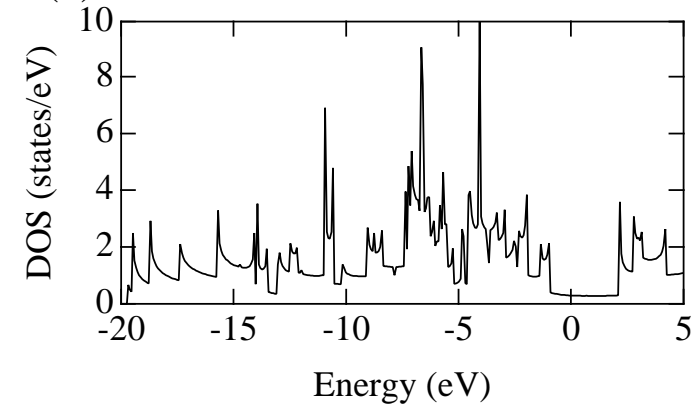

(c)

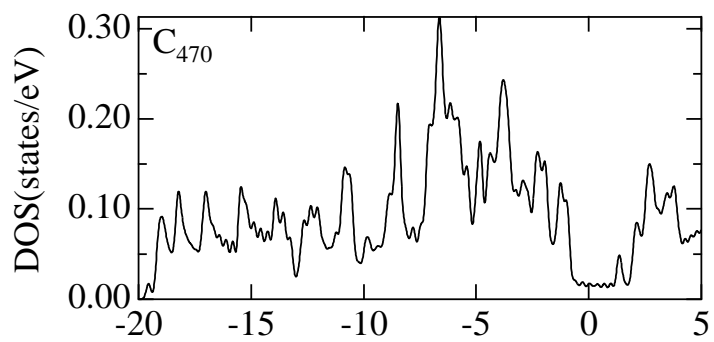

(d)

Energy (eV)

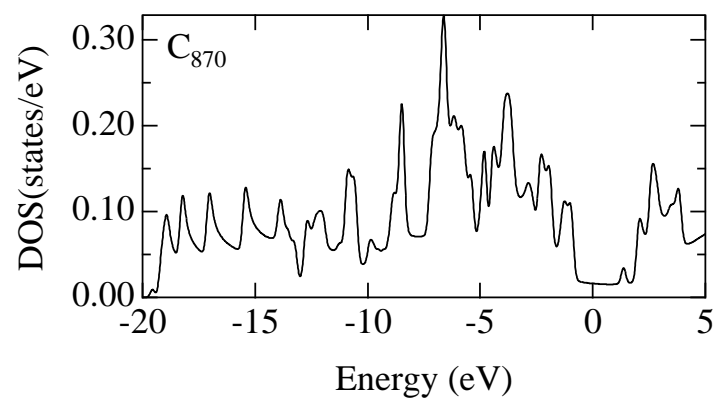

FIG. 2: DOS for capped $(5,5)$ nanotubes, (a) $\mathrm{C}_{90}$, (b) $\mathrm{C}_{290}$, (c) $\mathrm{C}_{470}$, and (d) $\mathrm{C}_{870}$. (e) DOS for the $(5,5)$ tube with infinite length. DOS are calculated per atom.

DOS for the finite-length $(5,5)$ nanotubes of which ends are capped by $\mathrm{C}_{60}$ hemispheres. The DOS is calculated by using a form $D(E)=\sum_{i} \frac{\Gamma}{\pi} \frac{1}{\left.\left(E-E_{i}\right)^{2}+\Gamma^{2}\right)}$ where $\Gamma$ is a broadening factor $(\Gamma=0.01 \mathrm{eV})$. In the tube with the short length, peaks of the DOS possess symmetric tails which are the characteristics of the zero-dimensionality of the system. In the tube with its length of $3.5 \mathrm{~nm}$ or longer, it is found that the asymmetric peak shape of the DOS exhibits one-dimensional character of the system. Furthermore, the finite and nearly constant DOS emerge around the Fermi energy and the characteristic peaks corresponding to the first gap of the metallic nanotubes emerge just above and below the flat DOS region. In addition to the characters caused by the nanotube, we also find that a peak around the energy of about $2 \mathrm{eV}$ is due to the electron states localized the pentagonal sites on the caps. The flatness of 
(a)

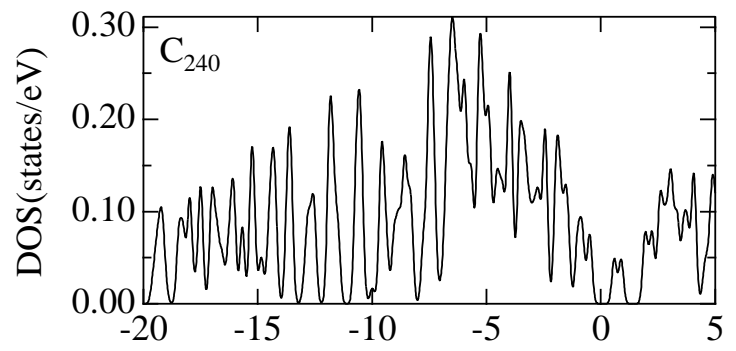

(b)

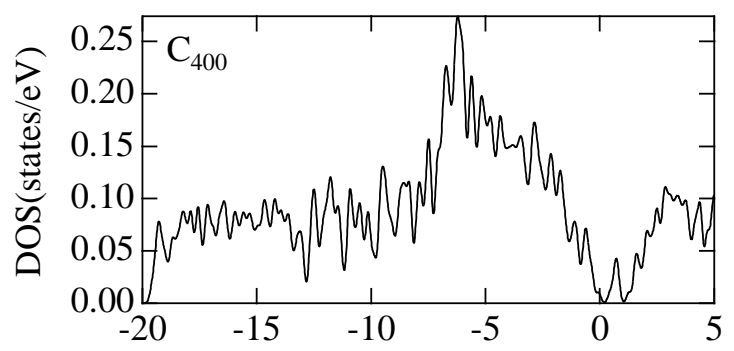

(e)

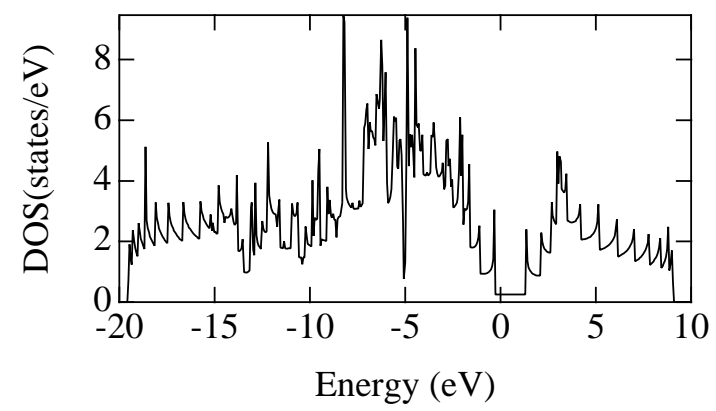

(c)

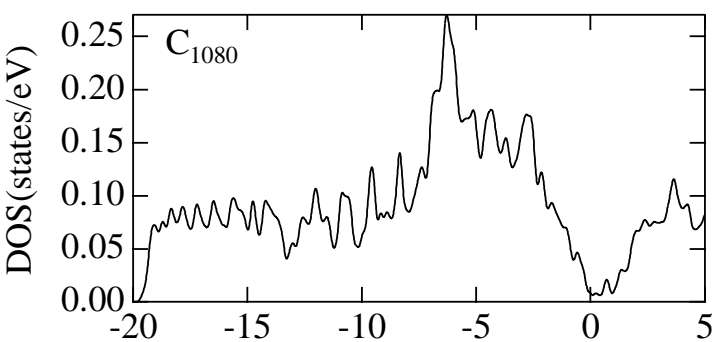

(d)

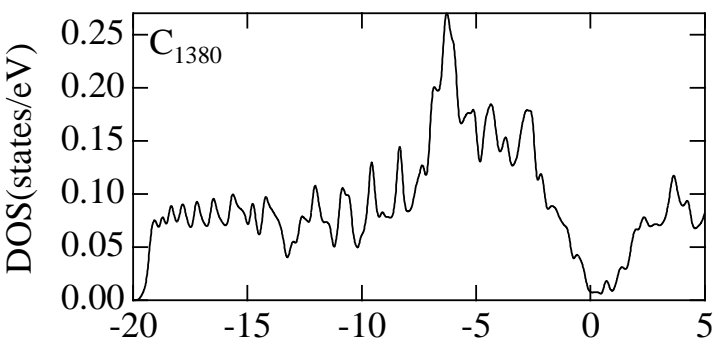

Energy (eV)

FIG. 3: DOS for capped $(10,10)$ nanotubes, (a) $\mathrm{C}_{240}$, (b) $\mathrm{C}_{400}$, (c) $\mathrm{C}_{1080}$, and (d) $\mathrm{C}_{1380}$. (e) DOS for the $(10,10)$ tube with infinite length. DOS are calculated per atom.

the DOS near the Fermi energy increases while the DOS peak originated from the pentagonal sites decreases with increasing the length of the tubular region. In particular, it is found that the electronic structure of the finite-length tube with $10 \mathrm{~nm}$ length experimentally observed exhibits almost the same as that of the tube with infinite length.

Figure 3 shows the DOS for the finite-length $(10,10)$ nanotubes of which ends are capped by the $\mathrm{C}_{240}$ hemispheres. In the length where DOS of the capped $(5,5)$ tube exhibits onedimensional character, capped $(10,10)$ tube still possesses symmetric DOS peaks representing its zero-dimensional character. The nanotube with its length of $6.6 \mathrm{~nm}$ eventually exhibits one-dimensional electronic properties with finite and nearly constant DOS around the Fermi level. The results show that the crossover point of the dimensionality strongly depends 
(a)

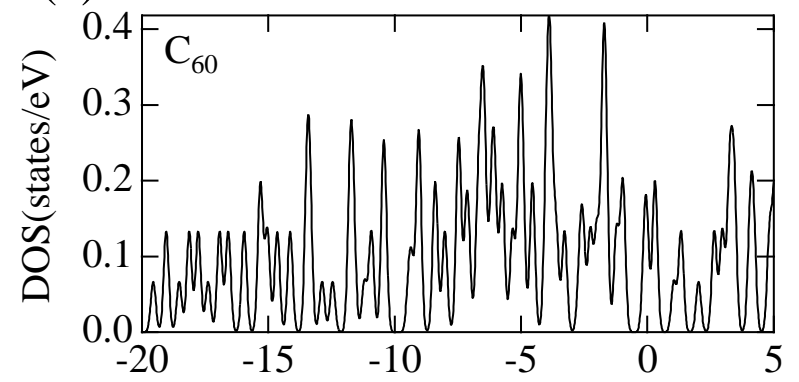

(b)

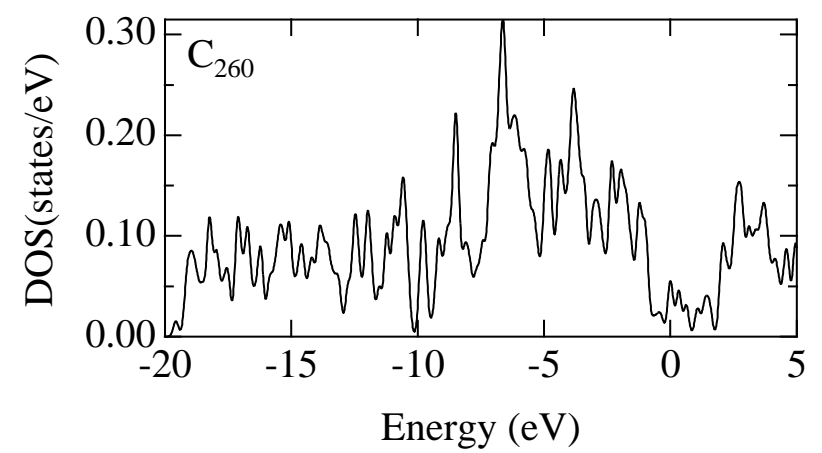

(c)

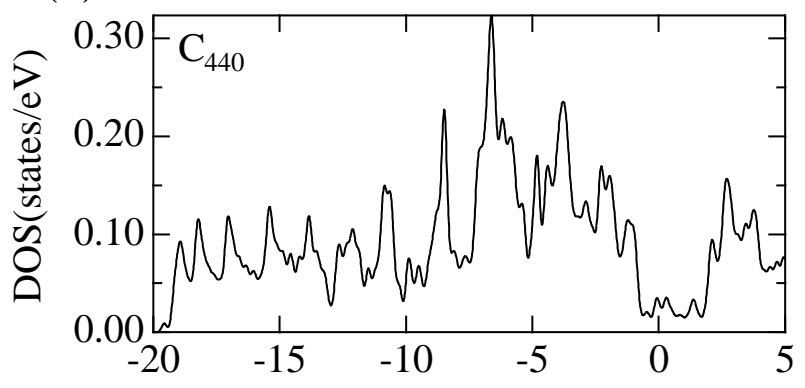

(d)

Energy (eV)

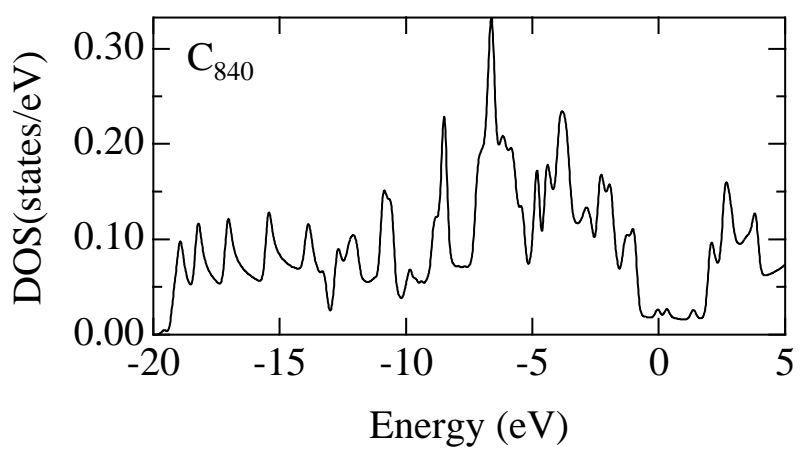

FIG. 4: DOS for $(5,5)$ nanotubes with cap-open ends, (a) $\mathrm{C}_{60}$, (b) $\mathrm{C}_{260}$, (c) $\mathrm{C}_{440}$, and (d) $\mathrm{C}_{840}$. DOS are calculated per atom.

on the length and the diameter of the nanotubes. However, we find that the aspect ratio that the tubes exhibit one-dimensional properties are universal for the tubes with various diameters: The calculated aspect ratio is found to be about 4 . The results also corroborate that the experimentally observed single-walled nanotubes with their length of $10 \mathrm{~nm}$ can be regarded as one-dimensional electron systems.

Finally, we show the electronic properties of the finite-length nanotube in which one of two ends is capped by the hemisphere of the $\mathrm{I}_{h}$ fullerenes while the other end is open possessing dangling bonds. The finite-length nanotubes with the capped-open structure are found to be the constituent unit of complexes in a sea-urchin shape consisting of a nanoparticle of the catalysts and the tubes. Figures 4 and 5 show the calculated DOS for the $(5,5)$ and $(10,10)$ nanotubes with capped-open end structure. In both tubes, we find that the tubes manifest their one-dimension character near the length where the capped tubes exhibit one-dimensional DOS. Lengths of the cylindrical region are 2.92 and $5.34 \mathrm{~nm}$ for $(5,5)$ and $(10,10)$ nanotubes, respectively. Thus, we pronounce that the dimensionality of the carbon 
(a)

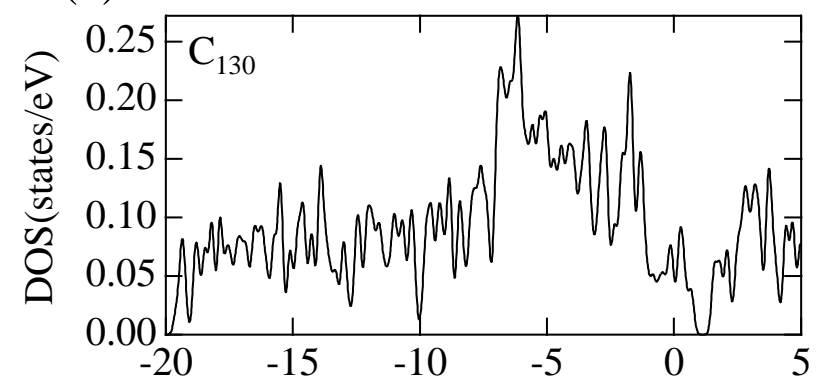

(b)

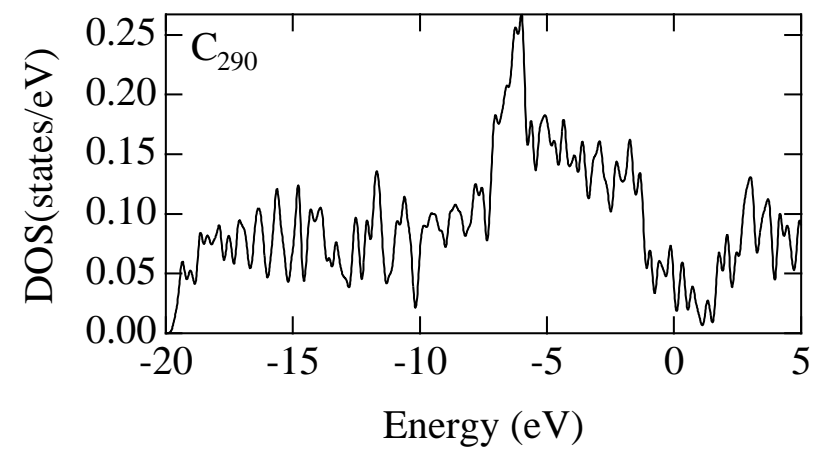

(c)

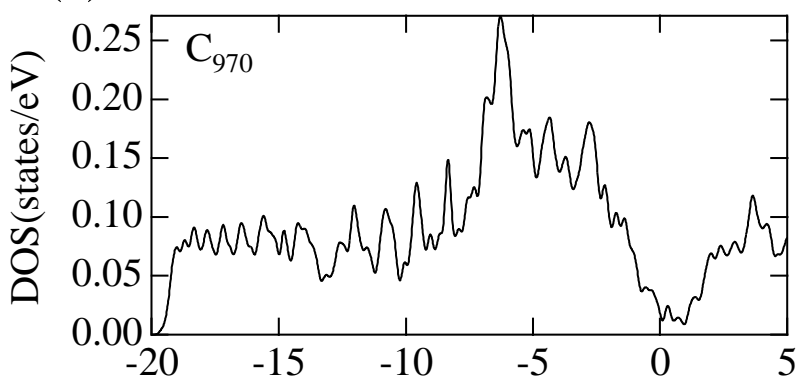

(d)

Energy $(\mathrm{eV})$

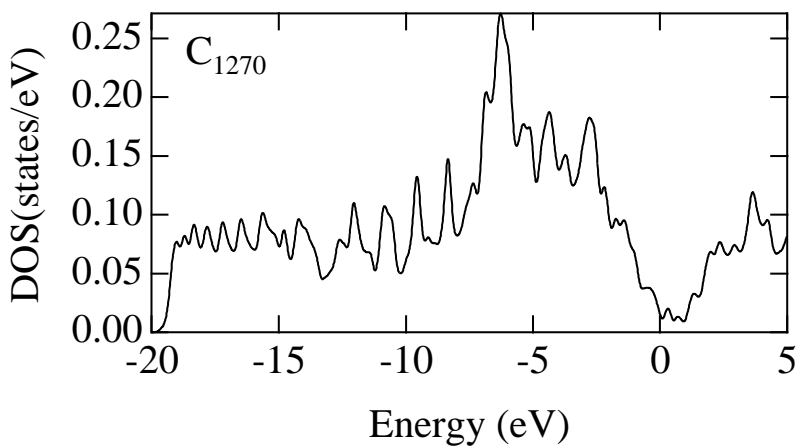

FIG. 5: DOS for $(10,10)$ nanotubes with cap-open ends, (a) $\mathrm{C}_{130}$, (b) $\mathrm{C}_{290}$, (c) $\mathrm{C}_{970}$, and (d) $\mathrm{C}_{1270}$. DOS are calculated per atom.

nanotubes with finite-length is determined by the aspect ratio of the tube diameter to the length of the cylindrical region. In the cap-open tubes, we find DOS peaks around $0 \mathrm{eV}$ which are caused by the dangling bond states besides structures near $2 \mathrm{eV}$ originated from the states localized on pentagonal sites. Both structures are found to decrease with increasing the length of the nanotubes since the effect of these sites on the DOS is negligible for the nanotubes with sufficient length.

\section{CONCLUSION}

Based on the generalized tight-binding calculations we explored the electronic structures of nanotubes with their length up to $10 \mathrm{~nm}$. We found that the electronic structures of the finite-length nanotubes experimentally observed $(10 \mathrm{~nm} \sim 100 \mathrm{~nm})$ are classified as the one-dimensional materials characterized by the anisotropic van Hove singularities. Our calculations have clarified that there are critical lengths in which the electronic properties of the finite-length nanotubes exhibit a crossover from one-dimensional character to the 
zero-dimensional one and vice versa. The length is determined by the aspect ratio of the tube diameter to the length of the cylindrical region. The calculated ratio is about 4 , which is common for all the nanotubes and independent from the end geometries. The present results corroborate that the nanotubes with their length of nano-meter scale is applicable for the nano-wires which connect the elements of nano-scale electronic devices.

\section{Acknowledgments}

We thank N. Hamada for providing the tight-binding program used in this work and also thank Y. Sato for providing their experimental results prior to publication. This work was partly supported by CREST-JST, Special Research Project on Nanoscience in University of Tsukuba and Grant-in-Aid for Scientific Research from Ministry of Education, Science, and Culture of Japan. Computations were done at Science Information Processing Center, University of Tsukuba, Yukawa Institute, Kyoto University, and at Research Center of the Computational Science, Okazaki National Institute.

[1] H. W. Kroto, J. R. Heath, S. C. O’Brien, R. F. Curl, and R. E. Smally, Nature, 318, 162 (1995).

[2] S. Iijima, Nature, 354, 56 (1991).

[3] For a review of early works on fullerene, see e.g. M. S. Dresselhaus, G. Dresselhaus, and P. C. Eklund, Science of Fullerenes and Carbon Nanotubes (Academic Press, San Diego, 1996).

[4] S. Saito, S. Okada, S. Sawada, and N. Hamada, Phys. Rev. Lett., 75, 685 (1995).

[5] N. Hamada, S. Sawada, and A. Oshiyama, Phys. Rev. Lett. 68, 1579 (1992).

[6] J. W. Mintmire, B. I. Dunlap, and C. T. White, Phys. Rev. Lett., 68, 631 (1992)

[7] R. Saito, M. Fujita, M. S. Dresselhaus, and G. Dresselhaus, Appl. Phys. Lett. 60, 2204 (1992).

[8] Y. Achiba, K. Kikuchi, Y. Aihara, T. Wakabayashi, Y. Miyake, and M. Kainosho, in "Science and Technology of Fullerene Mterials", eds. P. Bernier et al,, (Proc. Mat. Res. Soc. Vol. 359, Pittsburgh, 1995) p. 3.

[9] S. Bandow, M. Takizawa, K. Hirahara, M. Yudasaka and S. Iijima, Chme. Phys. Lett., 337 48 (2001). 
[10] Y. Sato, B. Jeyadevan, R. Hatakeyama, A. Kasuya, and K. Tohji, J. Phys. Chem. B, submitted.

[11] Y. Sato, private communications

[12] P. W. Fowler, J. Phys. Chem. Solids, 54, 1825 (1993).

[13] H. -Y. Zhu, D. J. Klein, T. G. Schmalz, A. Rubio, and N. H. March, J. Phys. Chem. Solids, 59, 417 (1998).

[14] R. A. Jishi, J. Bragin, and L. Lou, Phys. Rev. B 59, 9862 (1999).

[15] J. Cioslowski, N. Rao, and D. Moncrieff, J. Am. Chem. Soc., 124, 8485 (2002).

[16] Y. Nomura, H. Fujita, S. Narita, and T. Shibuya, Chem. Phys. Lett., 375, 72 (2003).

[17] S. Okada and A. Oshiyama, J. Phys. Soc. Japan, 72, 1510 (2003).

[18] Y. Matsuo, K. Tahara, and E. Nakamura, Organic Lett., 5, 3181 (2003).

[19] N. Hamada, unpublished.

[20] S. Okada and S. Saito, J. Phys. Soc. Japan, 64, 2100 (1995). 